\begin{tabular}{|c|c|}
\hline Title & p53 represses the transcription of snRNA genes by preventing the formation of little elongation complex \\
\hline Author(s) & $\begin{array}{l}\text { A nwar, Delnur; T akahashi, Hidehisa; W atanabe, Masashi; Suzuki, Masanobu; Fukuda, Satoshi; Hatakey ama, } \\
\text { Shigetsugu }\end{array}$ \\
\hline Citation & $\begin{array}{l}\text { Biochimica et biophy sica acta. Gene regulatory mechanisms, 1859(8), 975-982 } \\
\text { https://doi.org/10.1016/.bbagrm.2016.06.001 }\end{array}$ \\
\hline Issue Date & 2016-08 \\
\hline DOC URL & http:/hdl.handle.net/2115/66903 \\
\hline Rights & $\begin{array}{l}\text { (9 2016. This manuscript version is made available under the CC-BY-NC-ND } 4.0 \text { license } \\
\text { http://creativecommons.org/icenses/by-nc-nd/4.0/ }\end{array}$ \\
\hline Rights(URL) & https://creativecommons.org/icenses/by-nc-nd/4.0/ \\
\hline Type & article (author version) \\
\hline File Information & doi.org-10.1016-j.bbagrm.2016.06.001.pdf \\
\hline
\end{tabular}

Instructions for use 


\title{
p53 represses the transcription of $s n R N A$ genes by preventing the formation of little elongation complex
}

\author{
Delnur Anwar ${ }^{a, b}$, Hidehisa Takahashi ${ }^{a}$, Masashi Watanabe ${ }^{a}$, Masanobu Suzuki ${ }^{\text {a,b }}$, \\ Satoshi Fukuda ${ }^{\text {, }}$, Shigetsugu Hatakeyama, ${ }^{a, *}$
}

${ }^{a}$ Department of Biochemistry, Hokkaido University Graduate School of Medicine, Sapporo, Hokkaido 060-8638, Japan

${ }^{b}$ Department of Otolaryngology, Head \& Neck Surgery, Hokkaido University Graduate School of Medicine, Sapporo, Hokkaido 060-8638, Japan

*Corresponding author. Dr. Shigetsugu Hatakeyama, Department of Biochemistry, Hokkaido University Graduate School of Medicine, Kita 15 Nishi 7, Kita-ku, Sapporo, Hokkaido 060-8638, Japan

Tel: +81-11-706-5899; Fax: +81-11-706-5169

E-mail: hatas@med.hokudai.ac.jp

Keywords: p53, ELL, transcription, snRNA, LEC 


\begin{abstract}
The regulation of transcription by RNA polymerase II (Pol II) is important for a variety of cellular functions. ELL/EAF-containing little elongation complex (LEC) was found to be required for transcription of Pol II-dependent small nuclear RNA (snRNA) genes. It was shown that the tumor suppressor p53 interacts with ELL and inhibits transcription elongation activity of ELL. Here, we show that p53 inhibits interaction between ELL/EAF and ICE1 in LEC and thereby p53 represses transcription of Pol II-dependent snRNA genes through inhibiting LEC function. Furthermore, induction of p53 expression by ultraviolet (UV) irradiation decreases the occupancy of ICE1 at Pol II-dependent $s n R N A$ genes. Consistent with the results, knockdown of p53 increased both the expression of $\operatorname{snRNA}$ genes and the occupancy of Pol II and components of LEC at $\operatorname{sn} R N A$ genes. Our results indicate that p53 interferes with the interaction between ELL/EAF and ICE1 and represses transcription of $s n R N A$ genes by Pol II.
\end{abstract}




\section{Highlights}

- p53 interacts with ELL and inhibits transcription elongation activity of ELL.

- p53 inhibits interaction between ELL/EAF and ICE1 in LEC.

- p53 decreases the occupancy of ICE1 at Pol II-dependent snRNA genes.

- $\quad$ p53 represses transcription of $s n R N A$ genes. 


\section{Introduction}

Several regulatory systems of transcription by RNA polymerase II (Pol II) play important roles in many functions including signal transduction, cell proliferation/differentiation and development. Many genes including protein-coding genes and non-coding RNA (ncRNA) genes are transcribed by Pol II [1, 2]. The processes of gene transcription by Pol II basically consist of three steps: transcription initiation, elongation and termination. Many recent studies have shown that the process of transcription elongation has a crucial role in the regulation of gene expression. Transcription elongation factors including Eleven Lysine-rich Leukemia (ELL)/ ELL-associated factors (EAF) family members and positive transcription elongation factor $(\mathrm{P}-\mathrm{TEFb})$ restart the transcription of Pol II by repressing transient pausing [3]. It has been reported that two ELL/EAF-containing complexes, super elongation complex (SEC) and little elongation complex (LEC), specifically regulate the transcription of distinct genes $[4,5]$. LEC is composed of multiple subunits including the ELL/EAF component, KIAA0947, NARG2 and ZC3H8 [3, 6, 7]. KIAA0947 and NARG2 were also known as ICE1 and ICE2 (interacts with C-terminus of ELL subunits 1 and 2), respectively [4]. It has been shown that LEC plays an essential role in transcription of small nuclear RNA (snRNA) genes. Recently, we showed that human Mediator subunit MED26 contributes to the recruitment of LEC to a subset of $s n R N A$ genes [6]. ICE1 is one of the central components for the assembly of LEC [5]. It has been shown that LEC plays a role in both the transcription initiation and elongation phases of $s n R N A$ gene transcription [5]. However, the detailed function of ICE1 and ICE2 in LEC has not been elucidated. 
p53 is an important tumor suppressor that is involved in many processes of carcinogenesis in human cancers [8-12]. p53 directly binds to DNA and regulates gene expression to prevent mutations of the genome [13]. p53 is activated in response to alteration of normal cell homeostasis including DNA damage, nutrient starvation, heat shock, virus infection, $\mathrm{pH}$ change, hypoxia and oncogene activation $[14,15]$. In response to DNA damage, p53 is stabilized and acts as a transcription factor that directly regulates several hundred genes and indirectly more than one thousand genes [16]. p53 forms a tetramer on p53-responsive elements of target genes to induce or repress gene expression [17]. Expression of p53-targeted genes induced by exposure to ultraviolet (UV) irradiation include both proto-oncogenes and tumor suppressor genes. Mutations in the p53 gene are thought to be involved in the development of precancerous lesions in many types of human cancers. Although p53 directly regulates the transcription of protein-coding genes, recent studies have shown novel roles of p53 in regulation of the transcription of non-coding RNA genes. It has been reported that p53 represses the transcription of $s n R N A$ genes [18]. In addition, accumulating evidence indicates that snRNA plays a role in the carcinogenesis in lung cancer, germ cell tumors and several types of leukemia [19-21] and in the progression of human diseases including Alzheimer's disease [22-24].

It has been reported that p53 is one of the specific ELL interactors and that the C-terminal 47 amino acids of p53 are responsible for the interaction [25]. Intriguingly, p53 inhibits the transcription elongation activity of ELL [25]. These results raises the possibility that $\mathrm{p} 53$ regulates transcription elongation by Pol II through controlling the activity of ELL or LEC. Here, we provide evidence that p53 is involved in regulation of the function of LEC. Through biochemical analysis and chromatin immunoprecipitation 
(ChIP) analysis, we showed that p53 inhibits the interaction between ELL/EAF and ICE1 in LEC in vitro. Expression of p53 induced by UV irradiation decreased the occupancy of ICE1 at Pol II-dependent snRNA genes. p53 knockdown increases both the expression of $s n R N A$ genes and the occupancy of Pol II and the components of LEC at $s n R N A$ genes. These findings suggest that p53 interferes with the interaction between ELL/EAF and ICE1 and represses the transcription of $s n R N A$ genes by Pol II. 


\section{Materials and Methods}

\subsection{Cell culture}

HCT116 cells (ATCC CCL-247) and their derivatives were seeded and cultured in 10 -cm dishes $\left(2 \times 10^{6}\right.$ cells per dish) under an atmosphere of $5 \% \mathrm{CO}_{2}$ at $37^{\circ} \mathrm{C}$ in McCoy’s 5a Medium (GIBCO, Grand Island, NY) supplemented with 10\% (v/v) fetal bovine serum (Invitrogen, Carlsbad, CA), 100 units/ml penicillin, and $100 \mu \mathrm{g} / \mathrm{ml}$ streptomycin (GIBCO).

\subsection{Production of recombinant protein}

Epitope-tagged ELL, EAF1 and p53 were subcloned into pBacPAK8 and were expressed using the BacPAK system (Clontech Laboratories, Mountain View, CA). Full-length ICE1 was subcloned into pFastBac HTb with HA tags and expressed with the BAC-to-BAC system (Clontech). Baculovirus infections, Sf9 culture, and affinity purifications were performed as described previously [6].

\subsection{Antibodies and reagents}

Anti-FLAG (M2) antibody (F3165), anti-FLAG (M2)-agarose (A2220) and anti-HA-agarose (A2095) were purchased from Sigma-Aldrich (St. Louis, MO). Anti-ELL antibody (A301-645A) and anti-ICE1 antibody (A304-276A) were purchased from Bethyl Laboratories (Montgomery, TX). Anti-Pol II antibody (F-12, sc-55492), 
anti-p53 antibody (DO-1, ab1101), normal rabbit IgG (sc-2027) and normal mouse IgG (sc-2025) were purchased from Santa Cruz Biotechnology. Anti-Myc antibody (9E10, MMS-164P) and anti-HA antibody (HA11, 901502) were purchased from Covance Research Products (Princeton, NJ). Anti-TBP antibody (ab51841) was purchased from Abcam (Cambridge, MA). Probond resin was purchased from Life Technologies (Carlsbad, CA).

\subsection{Immunoprecipitation}

Sf9 cells $\left(3 \times 10^{7}\right.$ cells per 15 -cm dish $)$ or HCT116 cells $\left(1 \times 10^{7}\right.$ cells per $10-\mathrm{cm}$ dish) were lysed with $1 \mathrm{ml}$ of lysis buffer, and 3\% of whole cell lysates (WCL) in SDS sample buffer were boiled for $5 \mathrm{~min}$. For the remaining lysates, $5 \mu \mathrm{g}$ of an antibody was added and incubated for $1 \mathrm{~h}$ at $4^{\circ} \mathrm{C}$. Twenty $\mu$ of $50(\mathrm{v} / \mathrm{v}) \%$ protein A sepharose 4 fast flows were added and incubated for $2 \mathrm{~h}$ at $4^{\circ} \mathrm{C}$. After washing five times, immunoprecipitates were eluted with FLAG peptide (Sigma) or HA peptide (Sigma), and the resulting eluates $(0.01 \mathrm{mg} / \mathrm{ml})$ were subjected to SDS-PAGE.

\section{5. siRNA transfection and UV irradiation}

HCT116 cells were cultured in six-well tissue culture plates $\left(\sim 1 \times 10^{5}\right.$ cells per well) or 10 -cm dishes $\left(\sim 2 \times 10^{6}\right.$ cells per dish). The cells were transfected with $25 \mathrm{nM}$ siRNAs targeting p53 or $25 \mathrm{nM}$ siCONTROL NON-TARGETING siRNA using Lipofectamine RNAiMAX Transfection Reagent (Invitrogen). Cells were harvested at $48 \mathrm{~h}$ after transfection. Then the cells were treated with $20 \mathrm{~J}$ of UV (254-nm peak) $/ \mathrm{m}^{2}$ 
by CL-1000S UV Crosslinker (UVP, Upland, CA). After 1-h incubation, the cells were harvested.

\subsection{Gene expression analysis}

Total RNA was isolated using Isogen II (Nippon Gene, Tokyo, Japan). For RT-qPCR, 500 ng of total mRNA was reverse-transcribed using the iScript Select cDNA Synthesis Kit (Biorad, Hercules, CA). The threshold cycle $(\mathrm{Ct})$ values were determined by real-time PCR reactions using an Applied Biosystems StepOne Realtime PCR System and Power SYBR Green PCR Master Mix (Life Technologies) and normalized by subtracting the $\mathrm{Ct}$ value of the GAPDH gene from the $\mathrm{Ct}$ value of the respective gene $\left(\Delta \mathrm{Ct}=\mathrm{Ct}^{\mathrm{gene}}-\mathrm{Ct}^{\mathrm{GAPDH}}\right)$. The relative mRNA levels were then calculated using $2^{-\Delta \mathrm{Ct}}$. The primer sequences in this study were as follows: Human U1 snRNA, 5'-GGGAGATACCATGATCACGAAGGT-3' (forward) and 5'-ATGCAGTCGAGTTTCCCACA-3' (reverse); Human U2 snRNA, 5'-GTTTAATATCTGATACGTCCTCTATCC-3' ' (forward) and 5'-TCGATGCGTGGAGTGGAC-3' (reverse); Human U5A snRNA, 5'-ACTCTGGTTTCTCTTCAGATCGCA-3' 5'-CTTGCCAAAGCAAGGCCTCAAA-3' (reverse); Human U5B snRNA, 5'-ACTCTGGTTTCTCTTCAGATCGT-3' (forward) and 5'-CTTGTCGGAACAAGGCCTCAAA-3' (reverse); Human U6 snRNA, 5'-GCTCGCTTCGGCAGCACATATACTAA-3', (forward) and 5'-ACGAATTTGCGTGTCATCCTTGCG-3' (reverse); Human U11 snRNA, 5'-TTCTGTCGTGAGTGGCACACGTA-3') (forward) and 


\begin{tabular}{|c|c|}
\hline 5'-AACGATCACCAGCTGCCCAAATAC-3' & (reverse); \\
\hline 5'-TCGACAGTCAGCCGCATCTTCTTT-3' & (forward) \\
\hline
\end{tabular}

\subsection{ChIP assays}

Cells $\left(5 \times 10^{6}\right)$ were cultured in a $10-\mathrm{cm}$ dish and crosslinked with $2 \mathrm{mM}$ disuccinimidyl glutarate (DSG) crosslinker in PBS for $30 \mathrm{~min}$ and then $1 \%$ formaldehyde in PBS for $10 \mathrm{~min}$ at room temperature. The cells were harvested and lysed with $2 \mathrm{ml}$ of ChIP lysis buffer. Cell lysates $(1 \mathrm{mg} / \mathrm{ml})$ were sonicated with a Bioruptor 15 times for $30 \mathrm{sec}$ each time. After sonication, micrococcal nuclease (2910A, Takara Bio Inc, Tokyo, Japan) was added to digest DNA. SureBeads Protein G Magnetic beads (Biorad) and specific antibodies were added to the digested lysates and incubated for $2 \mathrm{~h}$ at $4^{\circ} \mathrm{C}$. Specific antibodies including $6 \mu \mathrm{g}$ of anti-ICE1 antibody (A304-276A, Bethyl Laboratories), $8 \mu \mathrm{g}$ of anti-ELL antibody (A301-645A, Bethyl Laboratories), $10 \mu \mathrm{g}$ of anti-p53 antibody (ab1101, Abcam), $6 \mu \mathrm{g}$ of anti-Pol II antibody (sc-55492, Santa Cruz Biotechnology) and $10 \mu \mathrm{g}$ of anti-TBP antibody (ab51841, Abcam) were used in the assays. The beads were washed twice with CHIP buffer containing $200 \mathrm{mM} \mathrm{KCl}, 2 \mathrm{mM} \mathrm{CaCl} 2$ and $50 \mathrm{mM}$ Tris- $\mathrm{HCl} \mathrm{pH}$ 8.0, twice with $500 \mathrm{mM}$ $\mathrm{KCl} \mathrm{CHIP}$ wash buffer and once with TE buffer. Bound complexes were eluted from the beads with $100 \mathrm{mM} \mathrm{NaHCO} 3$ and $1 \%$ SDS by incubating at $50^{\circ} \mathrm{C}$ for $30 \mathrm{~min}$. Crosslinking was reversed by overnight incubation at $65^{\circ} \mathrm{C}$. Immunoprecipitated DNA and input DNA were treated with RNase A and proteinase $\mathrm{K}$ by incubation at $45^{\circ} \mathrm{C}$. DNA was purified using the QIAquick PCR purification kit (28106, Qiagen, Valencia, 
CA) or MinElute PCR purification kit (28006, Qiagen). Immunoprecipitates and input were analyzed by quantitative PCR. The ChIP signal was normalized to input. Three biological replicates were performed for each experiment.

\subsection{Statistical analysis}

Student's $t$-test was used to determine the statistical significance of experimental data. 


\section{Results}

\subsection{EAF1 depletion decreases the occupancy of ICE1 at Pol II-dependent snRNA genes}

We previously showed that the EAF family member protein functions as an adaptor molecule to connect MED26 and LEC, that ICE1 functions as a core subunit of LEC and interacts with both ICE2 (NARG2) and ELL/EAF, and that the C-terminal fragment of ICE1 (1191-2266) is required for the formation of LEC [3, 6]. EAF1 enhances the activity of ELL for Pol II elongation [26]. At first, to examine whether EAF1 is crucial for the occupancy of ICE1 at Pol II-dependent $s n R N A$ genes, we performed ChIP analysis using HEK293T cells that were transfected with control small interfering RNA (siRNA) or EAF1 siRNA. EAF1 knockdown caused a significant decrease in the occupancy of ICE1 in $U 4 C$ and $U 5 B$ snRNA genes but not in the $U 5 B$ upstream region and the $U 6$ snRNA gene, which is transcribed by Pol III (Fig. 1A and B). These findings suggest that EAF1 functions as an adaptor molecule connecting LEC and MED26 at a subset of Pol II-dependent $s n R N A$ genes.

\section{2. p53 interferes with the interaction between ELL/EAF and ICE1}

It has been reported that the C-terminal region of p53 interacts with ELL and inhibits the transcriptional activity of ELL and that the amino acid region from 35 to 249 of ELL and the amino acid region from 346 to 393 of p53 are required for the interaction between p53 and ELL [25]. We previously showed that LEC is composed of EAF, ELL, ICE1 and ICE2 and that MED26 recruits LEC to snRNA genes through interaction with EAF1 [6]. The N-terminal domain of MED26 (1-113) directly binds to 
the C-terminal region (245-268) of human EAF1 [3, 6]. Previous biochemical analysis indicated that the ELL/EAF binds to the ICE1/ICE2 subcomplex in LEC through direct interaction between ELL and ICE1 [6], raising the possibility that p53 interacts with ELL and interferes with the interaction between ELL with ICE1. We therefore performed an in vitro binding assay. We expressed HA-ICE1, FLAG-ELL, Myc-EAF and His $_{6}-\mathrm{p} 53$ in the indicated combinations through a baculovirus expression system. HA-ICE1 co-purified with FLAG-ELL or FLAG-ELL/Myc-EAF; however, coexpression of His $_{6}$-p53 decreased the complex formation of HA-ICE1 and FLAG-ELL (Fig. 2). These findings suggest that p53 interacts with ELL and interferes with the binding of ELL to ICE1, raising the possibility that p53 inhibits the recruitment of LEC to MED26 and decreases the expression of $s n R N A$ genes.

\subsection{UV irradiation decreases the occupancy of ICE1 at Pol II-dependent snRNA genes}

It has been reported that $\mathrm{p} 53$ activated by DNA damage represses transcription of snRNA genes and that the C-terminus (301-393) of p53 is responsible for its repression activity [18]. It has also been reported that the region from 35 to 249 amino acids of ELL and the region from 346 to 393 amino acids of p53 are required for the interaction between them [25]. Since we showed that p53 inhibits the interaction of ELL with ICE1, we speculated that induction of p53 expression by UV irradiation affects the occupancy of ICE1 at Pol II-dependent snRNA genes, which are regulated by LEC. Therefore, we performed ChIP analysis after UV irradiation as a cellular stress using HCT116 cells, which are well-known to expresses wild-type p53 [27]. UV treatment decreased the occupancy of ICE1 at $\operatorname{Rn} R A$ genes (Fig. 3A and B). This finding suggests that p53 expression induced by UV irradiation inhibits the recruitment of LEC to target genes by 
inhibiting the interaction of ELL with ICE1.

\section{4. p53 knockdown increases the occupancy of Pol II, ICE1 and ELL at snRNA genes}

We next tested whether p53 knockdown affected the occupancy of Pol II and

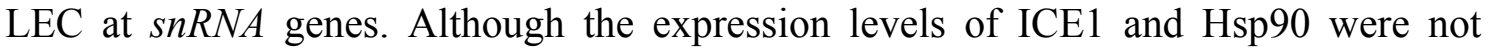
affected by UV irradiation and/or p53 knockdown, the expression level of p53 was increased by UV irradiation and was decreased by p53 knockdown (Fig. 4A). Consistent with our idea that p53 inhibits the recruitment of LEC at $s n R N A$ genes by interfering with the interaction of ELL with ICE1, p53 knockdown increased the amount of Pol II at snRNA genes including U4, U5B, U11 and $U 12$ (Fig. 4B). p53 knockdown also increased the occupancy of ICE1 and ELL at snRNA genes (Fig. 4C), but the occupancy of p53 was significantly decreased at $s n R N A$ genes including $U 4 C$, $U 5 B$ and $U 11$ genes in p53-knockdown cells (Supplementary Fig. 1 and 2). It has been reported that UV irradiation stimulated p53 association with $s n R N A$ genes and that $\mathrm{p} 53$ repressed transcription of human $s n R N A$ genes by Pol II [18]. To clarify the function of p53 in regulation of the transcription of snRNA genes, we performed quantitative real-time PCR analysis using p53-knockdown HCT116 cells treated with UV irradiation. After UV irradiation, p53 knockdown caused increased expression of $s n R N A$ genes including $U 4 C, U 5 B$ and $U 11$ (Fig. 5A). Furthermore, p53 knockdown increased the occupancy of ICE1 and Pol II at the U5B snRNA gene after UV irradiation (Fig. 5B).

It has been repored that human $s n R N A$ gene promoters contain a binding site(s) of p53 and that p53 is present at human $s n R N A$ gene promoters [18]. Since the tetramerization domain (326-356 amino acids) of $\mathrm{p} 53$ is responsible both for $\mathrm{p} 53$ 
binding to the promoters and for interaction with ELL $[18,28]$, we hypothesized that p53 binds to the promoter or to ELL in a mutually exclusive manner. Consistent with our idea, p53 occupancy was significantly decreased at U4C, U5A and U11 Pol II-dependent snRNA genes after UV irradiation (Supplementary Fig. 3). These findings indicate that $\mathrm{p} 53$ is released from the $s n R N A$ gene promoters after UV irradiation. Taken together, our results suggest that p53 dissociates from the promoters and then represses the expression of Pol II-dependent $s n R N A$ genes through inhibiting the formation of LEC at snRNA genes (Fig. 6). 


\section{Discussion}

In this study, we showed that EAF1 is required for the recruitment of ICE1 to a subset of RNA Pol II-dependent $s n R N A$ genes. We provided a putative model showing that EAF1 functions as an adaptor molecule connecting LEC and MED26 at a subset of Pol II-dependent snRNA genes (Fig. 6). According to our model, p53 interacts with ELL and inhibits the interaction between ELL and ICE1, leading to repression of the formation of LEC at $s n R N A$ genes. Expression of p53 induced by UV irradiation decreased the occupancy of ICE1 at Pol II-dependent snRNA genes. Moreover, p53 repressed the occupancy of not only Pol II but also ICE1 and ELL at $s n R N A$ genes, resulting in downregulation of Pol II-dependent $s n R N A$ genes. Taken together, p53 likely decreases the transcription of a subset of Pol II-dependent $s n R N A$ genes via interaction with ELL.

It has been reported that p53 also interacts with the key transcription elongation factor, $\mathrm{p}-\mathrm{TEFb}$, which is a component of SEC, and that SEC is required for proper induction of the HSP70 gene upon heat stress and is involved in HIV proviral transcription [29]. SEC plays a role in the proper coordinated induction of Hox genes during early developmental stages and is also involved in the misregulation of Hox genes in leukemia [30-34]. If p53 can also interact with ELL in SEC, it may inhibit the formation of SEC as well. This raises the possibility that p53 plays a role in regulation of the expression of target genes of SEC by inhibiting the recruitment of SEC. Importantly, it has been shown that SEC is required for $c-M y c$ expression in several leukemia cell lines, suggesting that SEC is a potential therapeutic target for the treatment of leukemia and other solid cancers associated with overexpression of the 
$c-M y c$ gene [35]. p53 has also been shown to repress the expression of $c-M y c$ [36]; however, the mechanism by which p53 regulates the expression of $c-M y c$ has not been elucidated. Our results suggest that p53 plays a crucial role in the inhibition of $c-M y c$ expression by inhibiting the interaction of ELL with other components of SEC.

The N-terminal domain of MED26 contributes to the recruitment of SEC to Pol II-transcribed protein-coding genes and to the recruitment of LEC to $s n R N A$ genes through direct interaction with EAF and likely helps to release Pol II from pausing [3, 6]. Several studies have shown that transcriptional regulation of $s n R N A$ genes is involved in the carcinogenesis of several types of leukemia, lung cancer and germ cell tumors [19-21]. Our results raise the possibility that p53 inhibits transcription of $s n R N A$ genes through inhibiting the recruitment of LEC to MED26 and functions as an oncosuppressor.

Taken together, the results indicate that p53 regulates transcription of Pol II-dependent $s n R N A$ genes through inhibiting the function of LEC. The p53 gene has been shown to be mutated or lost in more than $50 \%$ of cancers. Therefore, the results of our study suggest that $\mathrm{p} 53$ regulates the transcription of $s n R N A$ genes through inhibiting transcription elongation factors such as ELL/EAF and/or LEC, and the results may contribute to an understanding of diseases associated with dysregulation of $\operatorname{sn} R N A$ gene transcription. 


\section{Acknowledgements}

We would like to thank Drs. Joan W. Conaway and Ronald C. Conaway (Stowers Institute for Medical Research) for materials and Dr. Alijan Kadir for helpful discussion. We are grateful to Ms. Miho Uchiumi for help in preparing the manuscript and Ms. Nozomi Sakurai for technical assistance. This work was supported in part by KAKENHI (15H04690 and 24112006 to S. Hatakeyama; 15H04701, 26670130, and 24118002 to H. Takahashi) from the Ministry of Education, Culture, Sports, Science and Technology in Japan and by the Suhara Memorial Foundation (to S. Hatakeyama), Takeda Science Foundation (to H. Takahashi), Mochida Memorial Foundation (to H. Takahashi), Uehara Memorial Foundation (to H. Takahashi) and Senshin Medical Research Foundation (to H. Takahashi). 


\section{References}

[1] A.G. Matera, R.M. Terns, M.P. Terns, Non-coding RNAs: lessons from the small nuclear and small nucleolar RNAs, Nat Rev Mol Cell Biol, 8 (2007) 209-220.

[2] J.A. Goodrich, J.F. Kugel, Non-coding-RNA regulators of RNA polymerase II transcription, Nat Rev Mol Cell Biol, 7 (2006) 612-616.

[3] H. Takahashi, T.J. Parmely, S. Sato, C. Tomomori-Sato, C.A. Banks, S.E. Kong, H. Szutorisz, S.K. Swanson, S. Martin-Brown, M.P. Washburn, L. Florens, C.W. Seidel, C. Lin, E.R. Smith, A. Shilatifard, R.C. Conaway, J.W. Conaway, Human mediator subunit MED26 functions as a docking site for transcription elongation factors, Cell, 146 (2011) 92-104.

[4] Edwin R. Smith, C. Lin, Alexander S. Garrett, J. Thornton, N. Mohaghegh, D. Hu, J. Jackson, A. Saraf, Selene K. Swanson, C. Seidel, L. Florens, Michael P. Washburn, Joel C. Eissenberg, A. Shilatifard, The Little Elongation Complex Regulates Small Nuclear RNA Transcription, Mol Cell, 44 (2011) 954-965.

[5] D. Hu, E.R. Smith, A.S. Garruss, N. Mohaghegh, J.M. Varberg, C. Lin, J. Jackson, X. Gao, A. Saraf, L. Florens, M.P. Washburn, J.C. Eissenberg, A. Shilatifard, The little elongation complex functions at initiation and elongation phases of snRNA gene transcription, Mol Cell, 51 (2013) 493-505.

[6] H. Takahashi, I. Takigawa, M. Watanabe, D. Anwar, M. Shibata, C. Tomomori-Sato, S. Sato, A. Ranjan, C.W. Seidel, T. Tsukiyama, W. Mizushima, M. Hayashi, Y. Ohkawa, J.W. Conaway, R.C. Conaway, S. Hatakeyama, MED26 regulates the transcription of snRNA genes through the recruitment of little elongation complex, Nat Commun, 6 (2015) 5941. 
[7] D. Hu, Edwin R. Smith, Alexander S. Garruss, N. Mohaghegh, Joseph M. Varberg, C. Lin, J. Jackson, X. Gao, A. Saraf, L. Florens, Michael P. Washburn, Joel C. Eissenberg, A. Shilatifard, The Little Elongation Complex Functions at Initiation and Elongation Phases of snRNA Gene Transcription, Mol Cell, 51 (2013) 493-505.

[8] G. Matlashewski, P. Lamb, D. Pim, J. Peacock, L. Crawford, S. Benchimol, Isolation and characterization of a human p53 cDNA clone: expression of the human p53 gene, EMBO J, 3 (1984) 3257-3262.

[9] M. Isobe, B.S. Emanuel, D. Givol, M. Oren, C.M. Croce, Localization of gene for human p53 tumour antigen to band 17p13, Nature, 320 (1986) 84-85.

[10] S.E. Kern, K.W. Kinzler, A. Bruskin, D. Jarosz, P. Friedman, C. Prives, B. Vogelstein, Identification of p53 as a sequence-specific DNA-binding protein, Science, 252 (1991) 1708-1711.

[11] O.W. McBride, D. Merry, D. Givol, The gene for human p53 cellular tumor antigen is located on chromosome 17 short arm (17p13), Proc Natl Acad Sci USA, 83 (1986) 130-134.

[12] J.C. Bourdon, K. Fernandes, F. Murray-Zmijewski, G. Liu, A. Diot, D.P. Xirodimas, M.K. Saville, D.P. Lane, p53 isoforms can regulate p53 transcriptional activity, Genes Dev, 19 (2005) 2122-2137.

[13] D. Lane, A. Levine, p53 Research: the past thirty years and the next thirty years, Cold Spring Harb Perspect Biol, 2 (2010) a000893.

[14] M. Oren, Decision making by p53: life, death and cancer, Cell Death Differ, 10 (2003) 431-442.

[15] F. Su, L.E. Pascal, W. Xiao, Z. Wang, Tumor suppressor U19/EAF2 regulates 
thrombospondin-1 expression via p53, Oncogene, 29 (2010) 421-431.

[16] S. Chikayama, T. Sugano, Y. Takahashi, M. Ikeda, S. Kimura, Y. Kobayashi, M. Kondo, Nuclear accumulation of p53 protein and apoptosis induced by various anticancer agents, u.v.-irradiation and heat shock in primary normal human skin fibroblasts, Int J Oncol, 16 (2000) 1117-1124.

[17] S. Surget, M.P. Khoury, J.C. Bourdon, Uncovering the role of p53 splice variants in human malignancy: a clinical perspective, Onco Targets Ther, 7 (2013) 57-68.

[18] A.A. Gridasova, R.W. Henry, The p53 tumor suppressor protein represses human snRNA gene transcription by RNA polymerases II and III independently of sequence-specific DNA binding, Mol Cell Biol, 25 (2005) 3247-3260.

[19] J. Köhler, M. Schuler, T.C. Gauler, S. Nöpel-Dünnebacke, M. Ahrens, A.-C. Hoffmann, S. Kasper, F. Nensa, B. Gomez, M. Hahnemann, F. Breitenbuecher, D. Cheufou, F. Özkan, K. Darwiche, M. Hoiczyk, H. Reis, S. Welter, W.E.E. Eberhardt, M. Eisenacher, H. Teschler, G. Stamatis, W. Schmiegel, S.A. Hahn, A. Baraniskin, Circulating U2 small nuclear RNA fragments as a diagnostic and prognostic biomarker in lung cancer patients, J Cancer Res Clin Oncol, 142 (2016) 795-805.

[20] J.E. Holt, S.J. Stanger, B. Nixon, E.A. McLaughlin, Non-coding RNA in Spermatogenesis and Epididymal Maturation, in: D. Wilhelm, P. Bernard (Eds.) Non-coding RNA and the Reproductive System, Springer Netherlands, Dordrecht, 2016, pp. 95-120.

[21] R.A. Padgett, New connections between splicing and human disease, Trends Genet, 28 (2012) 147-154.

[22] Y. Jia, John C. Mu, Susan L. Ackerman, Mutation of a U2 snRNA Gene Causes 
Global Disruption of Alternative Splicing and Neurodegeneration, Cell, 148 (2012) 296-308.

[23] C.M. Hales, E.B. Dammer, I. Diner, H. Yi, N.T. Seyfried, M. Gearing, J.D. Glass, T.J. Montine, A.I. Levey, J.J. Lah, Aggregates of Small Nuclear Ribonucleic Acids (snRNAs) in Alzheimer's Disease, Brain Pathol, 24 (2014) 344-351.

[24] B. Bai, C. Hales, P. Chen, Y. Gozal, E. Dammer, J. Fritz, X. Wang, Q. Xia, D. Duong, C. Street, G. Cantero, D. Cheng, D. Jones, Z. Wu, Y. Li, I. Diner, C. Heilman, H. Rees, H. Wu, L. Lin, K. Szulwach, M. Gearing, E. Mufson, D. Bennett, T. Montine, N. Seyfried, T. Wingo, Y. Sun, P. Jin, J. Hanfelt, U1 small nuclear ribonucleoprotein complex and RNA splicing alterations in Alzheimer's disease, Proc Natl Acad Sci USA, 110 (2013) 16562-16567.

[25] N. Shinobu, T. Maeda, T. Aso, T. Ito, T. Kondo, K. Koike, M. Hatakeyama, Physical Interaction and Functional Antagonism between the RNA Polymerase II Elongation Factor ELL and p53, J Biol Chem, 274 (1999) 17003-17010.

[26] S.E. Kong, C.A. Banks, A. Shilatifard, J.W. Conaway, R.C. Conaway, ELL-associated factors 1 and 2 are positive regulators of RNA polymerase II elongation factor ELL, Proc Natl Acad Sci USA, 102 (2005) 10094-10098.

[27] M.D. Kaeser, S. Pebernard, R.D. Iggo, Regulation of p53 Stability and Function in HCT116 Colon Cancer Cells, J Biol Chem, 279 (2004) 7598-7605.

[28] R. Kamada, T. Nomura, C.W. Anderson, K. Sakaguchi, Cancer-associated p53 tetramerization domain mutants: quantitative analysis reveals a low threshold for tumor suppressor inactivation, J Biol Chem, 286 (2011) 252-258.

[29] P.P. Claudio, J. Cui, M. Ghafouri, C. Mariano, M.K. White, M. Safak, J.B. Sheffield, A. Giordano, K. Khalili, S. Amini, B.E. Sawaya, Cdk9 phosphorylates 
p53 on serine 392 independently of CKII, J Cell Physiol, 208 (2006) 602-612.

[30] N. He, M. Liu, J. Hsu, Y. Xue, S. Chou, A. Burlingame, N.J. Krogan, T. Alber, Q. Zhou, HIV-1 Tat and host AFF4 recruit two transcription elongation factors into a bifunctional complex for coordinated activation of HIV-1 transcription, Mol Cell, 38 (2010) 428-438.

[31] C. Lin, A.S. Garrett, B. De Kumar, E.R. Smith, M. Gogol, C. Seidel, R. Krumlauf, A. Shilatifard, Dynamic transcriptional events in embryonic stem cells mediated by the super elongation complex (SEC), Genes Dev, 25 (2011) 1486-1498.

[32] C. Lin, E.R. Smith, H. Takahashi, K.C. Lai, S. Martin-Brown, L. Florens, M.P. Washburn, J.W. Conaway, R.C. Conaway, A. Shilatifard, AFF4, a component of the ELL/P-TEFb elongation complex and a shared subunit of MLL chimeras, can link transcription elongation to leukemia, Mol Cell, 37 (2010) 429-437.

[33] B. Sobhian, N. Laguette, A. Yatim, M. Nakamura, Y. Levy, R. Kiernan, M. Benkirane, HIV-1 Tat assembles a multifunctional transcription elongation complex and stably associates with the 7SK snRNP, Mol Cell, 38 (2010) 439-451.

[34] A. Yokoyama, M. Lin, A. Naresh, I. Kitabayashi, M.L. Cleary, A higher-order complex containing AF4 and ENL family proteins with P-TEFb facilitates oncogenic and physiologic MLL-dependent transcription, Cancer Cell, 17 (2010) 198-212.

[35] Z. Luo, C. Lin, E. Guest, A.S. Garrett, N. Mohaghegh, S. Swanson, S. Marshall, L. Florens, M.P. Washburn, A. Shilatifard, The Super Elongation Complex Family of RNA Polymerase II Elongation Factors: Gene Target Specificity and Transcriptional Output, Mol Cell Biol, 32 (2012) 2608-2617.

[36] M. Sachdeva, S. Zhu, F. Wu, H. Wu, V. Walia, S. Kumar, R. Elble, K. Watabe, 
Y.-Y. Mo, p53 represses c-Myc through induction of the tumor suppressor miR-145, Proc Natl Acad Sci USA, 106 (2009) 3207-3212. 


\section{Figure legends}

Fig. 1. EAF1 is required for the occupancy of ICE1 at $s n R N A$ genes. (A) Occupancy of ICE1 at regions of $U 4 C, U 5 B$, upstream of $U 5 B$, and $U 6 s n R N A$ genes by EAF1 knockdown (KD). Standard deviations of each result were calculated from three independent experiments. $P$ values were determined by Student's $t$ test $(* P<0.05)$. Error bars show s.d. NS, not specific. (B) Western blot analysis of EAF1 after EAF1 knockdown. Hsp90 was used as a control.

Fig. 2. p53 interferes with the interaction between ELL/EAF and ICE1. Anti-FLAG-immunoprecipitates using lysates from Sf9 cells expressing the indicated combinations of Myc-tagged EAF, FLAG-tagged ELL, HA-tagged ICE1 and His $_{6}$-tagged p53 were subjected to immunoblot analysis. IP, immunprecipitation.

Fig. 3. UV irradiation decreases the occupancy of ICE1 at Pol II-dependent $s n R N A$ genes. (A) ChIP assays were performed using cells treated with or without UV irradiation. The occupancy of ICE1 at Pol II-dependent snRNA genes including $U 4 C$, $U 5 A$ and $U 11$ snRNA genes was quantified after UV irradiation. Standard deviations of each result were calculated from three independent experiments. $P$ values were determined by Student's $t$ test $(* P<0.1, * * P<0.05)$. Error bars show s.d. (B) Western blot analysis of p53 and ICE1 after UV irradiation using HCT116 cells. Hsp90 was used as a control.

Fig. 4. Knockdown of p53 increases the amounts of Pol II, ELL and ICE1 at a subset of 
snRNA genes. (A) Western blot analysis of p53 and ICE1 in p53-knockdown cells with or without UV irradiation. Hsp90 was used as a control. (B) The occupancy of Pol II at snRNA genes including $U 4 C, U 5 B, U 11$ and $U 12$ genes was analyzed using p53-knockdown (KD) cells. (C) The occupancy of ICE1 and ELL at snRNA genes including $U 5 A$ and $U 11$ genes was analyzed using p53-knockdown (KD) cells. Standard deviations of each result were calculated from three independent experiments. $P$ values were determined by Student's $t$ test $\left(* P<0.1,{ }^{*} P<0.05\right.$, *** $\left.P<0.01\right)$. Error bars show s.d.

Fig. 5. Knockdown of p53 increases in the expression of snRNA genes and the occupancy of ICE1 and Pol II after UV irradiation. (A) After UV irradiation, the expression of $s n R N A$ genes in p53-knockdown cells was analyzed by qPCR. (B) After UV irradiation, the occupancy of ICE1 and Pol II at the Pol II-dependent U5B snRNA gene was analyzed using p53-knockdown (KD) cells. Standard deviations of each result were calculated from three independent experiments. $P$ values were determined by Student's $t$ test (* $P<0.1, * * P<0.05)$. Error bars show s.d.

Fig. 6. Model for inhibition of the transcription of a subset of Pol II-dependent $s n R N A$ genes by p53. p53 represses the occupancy of ICE1 at $s n R N A$ genes, resulting in downregulation of Pol II-dependent snRNA genes through inhibiting the formation of LEC at $S n R N A$ genes. 


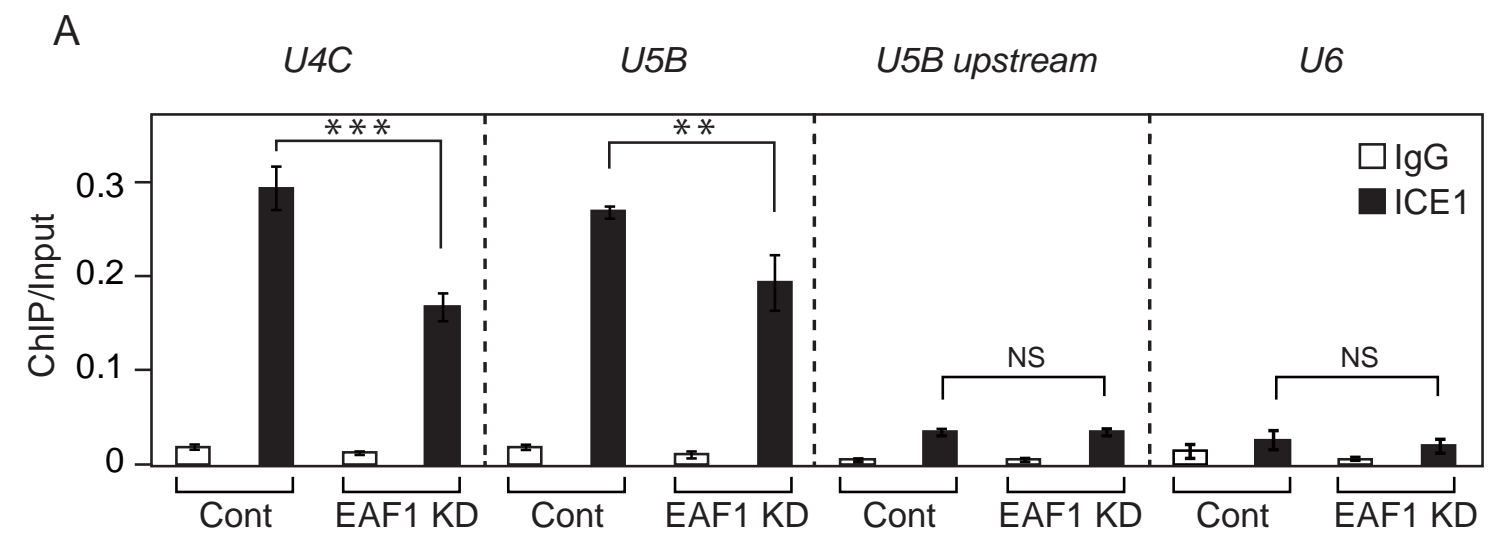

B
EAF1 SiRNA: $\quad-\quad+$
IB: anti-EAF1 $\square=E A F 1$
IB: anti-HSP90 - HSP90

Figure 1 


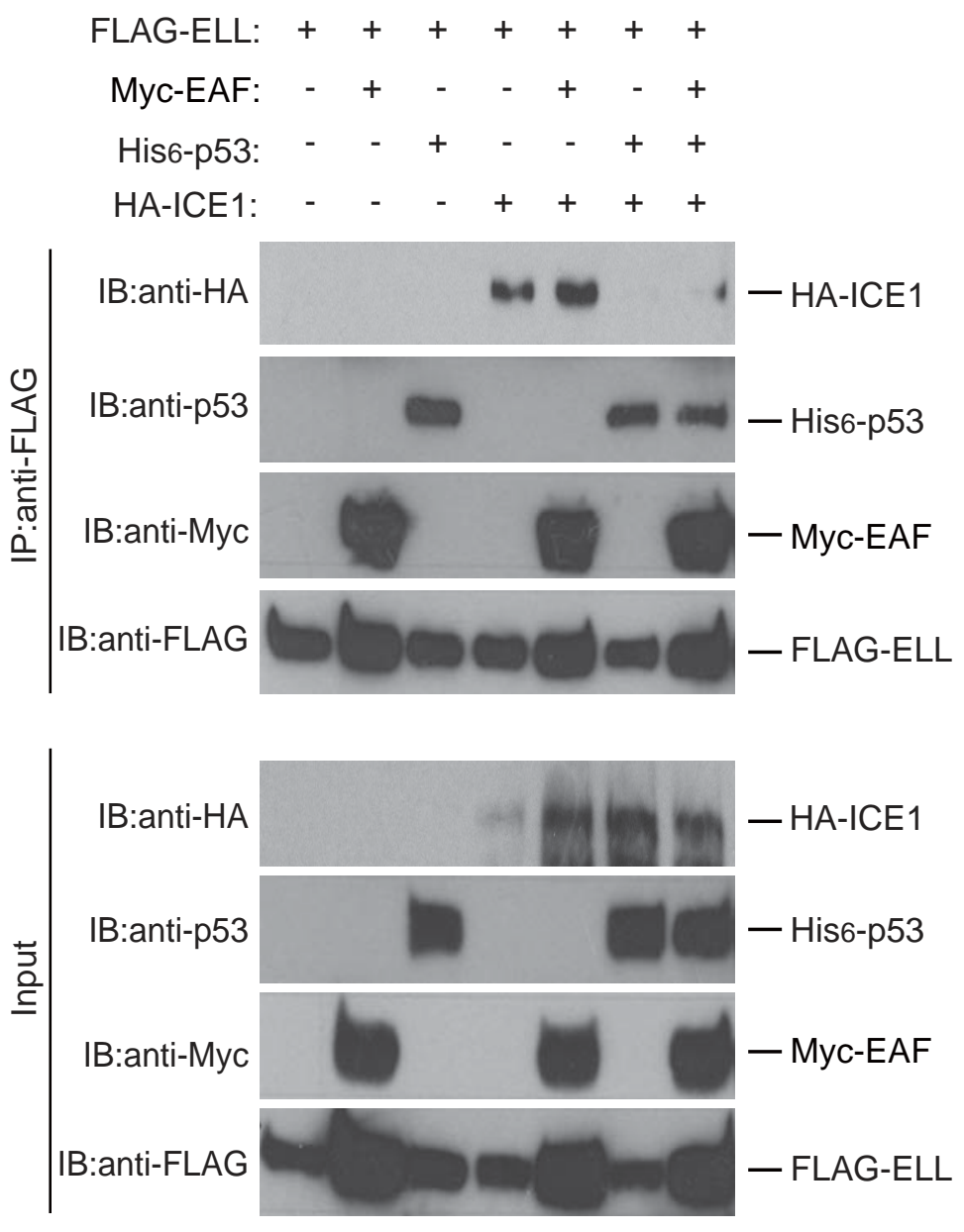

Figure 2 


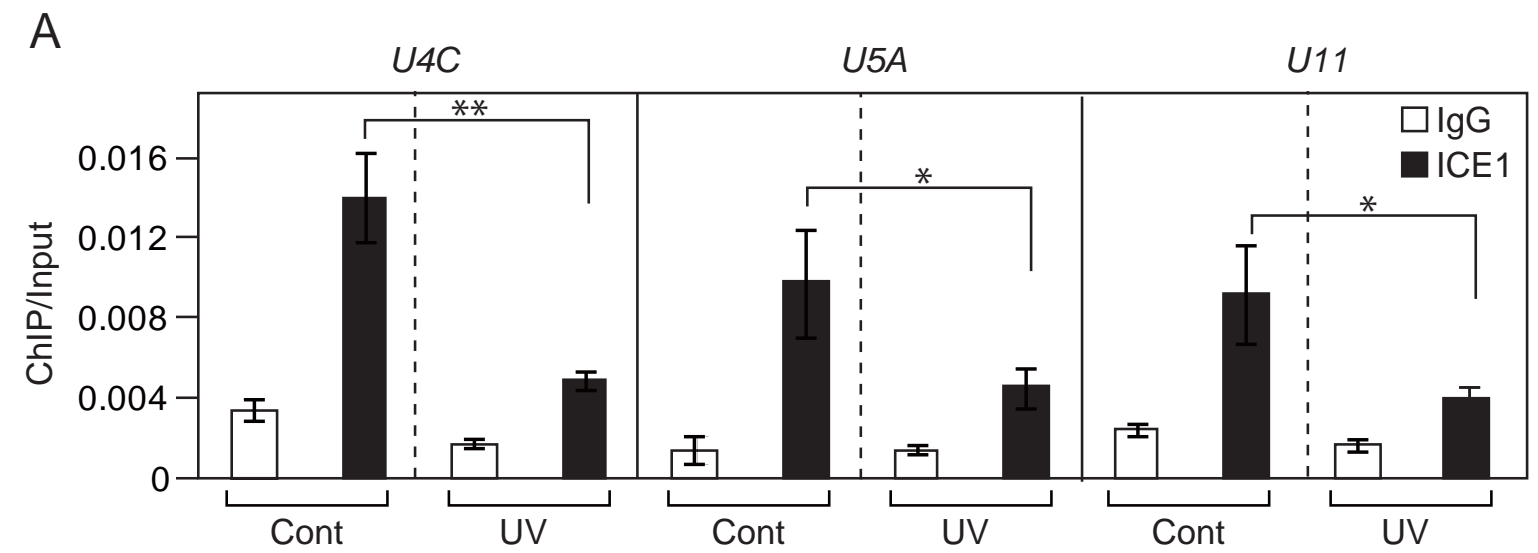

B
UV: $\quad-\quad+$
IB: anti-p53

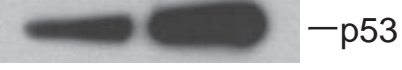
IB: anti-ICE1
IB: anti-HSP90
- ICE1
- HSP90

Figure 3 
A
UV: $-\quad-++$
p53siRNA: -+-+
IB: anti-p53
$-p 53$
IB: anti-ICE1
- -1 -ICE1
IB: anti-HSP90
-HSP90

B
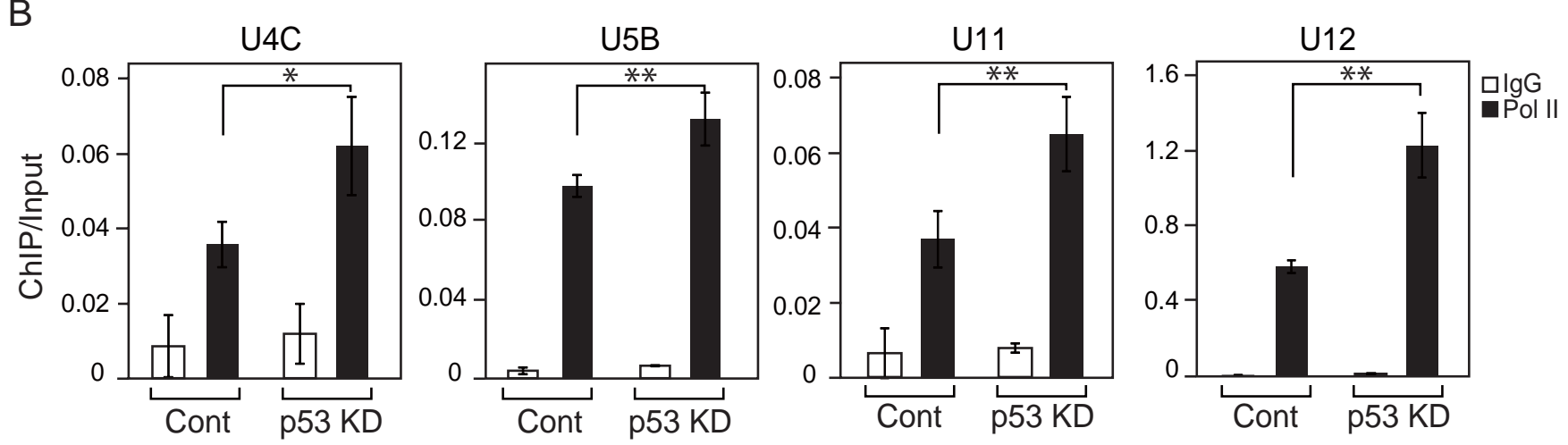

C
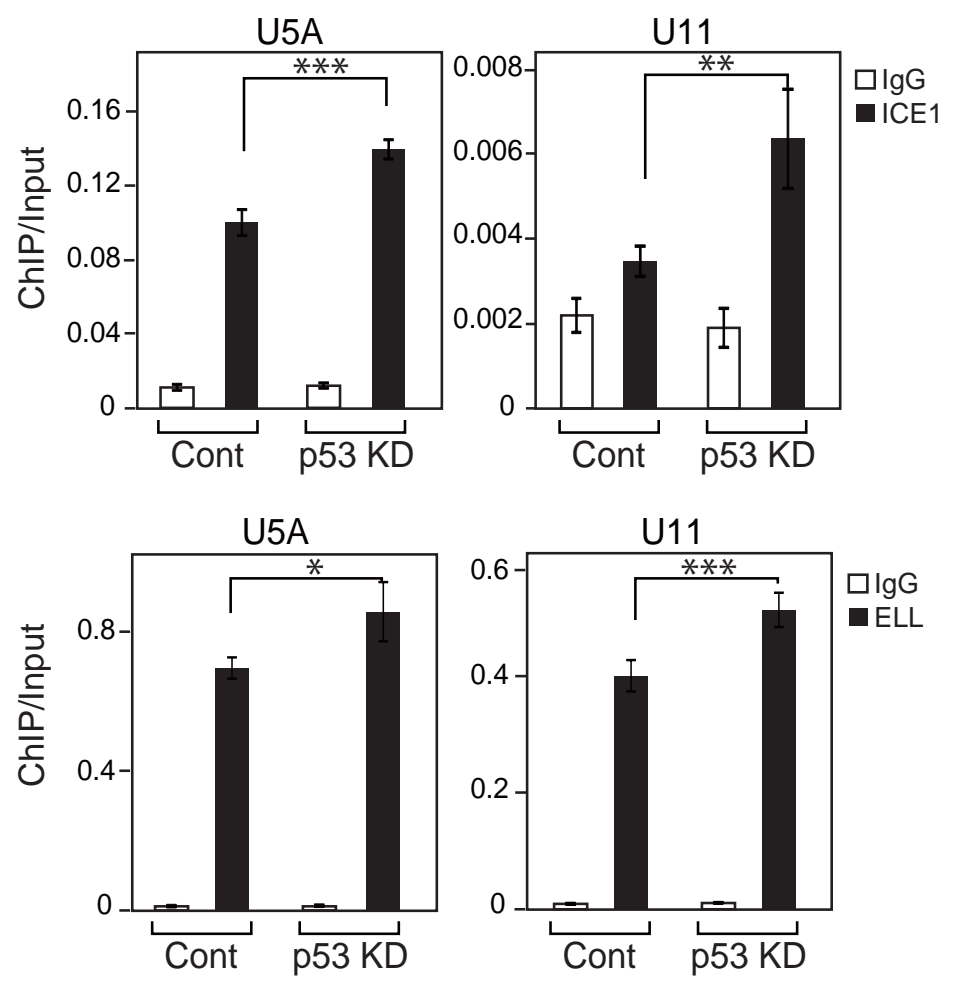

Figure 4 

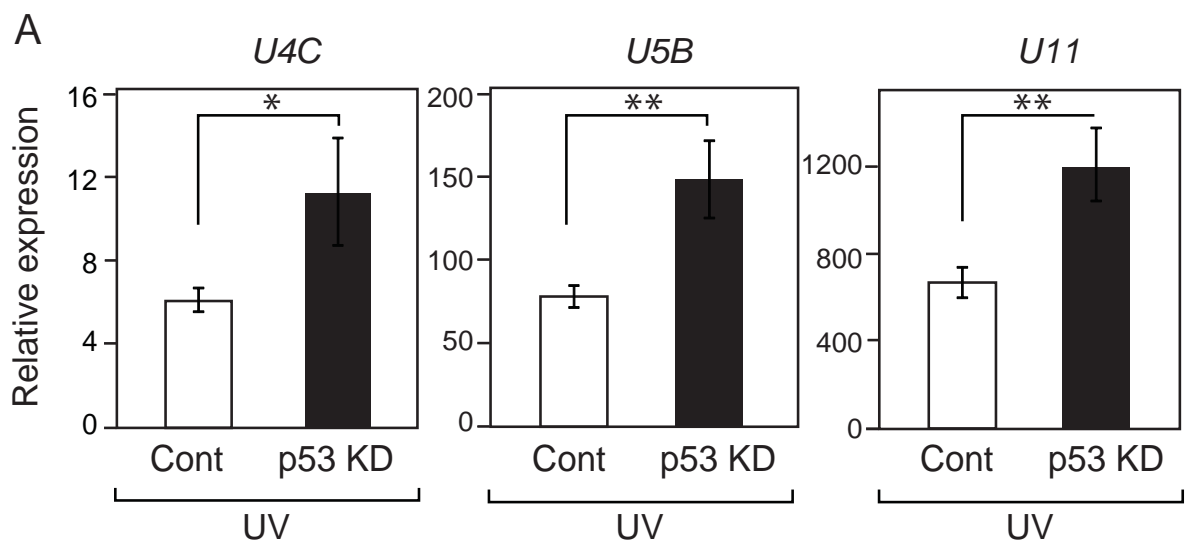

B
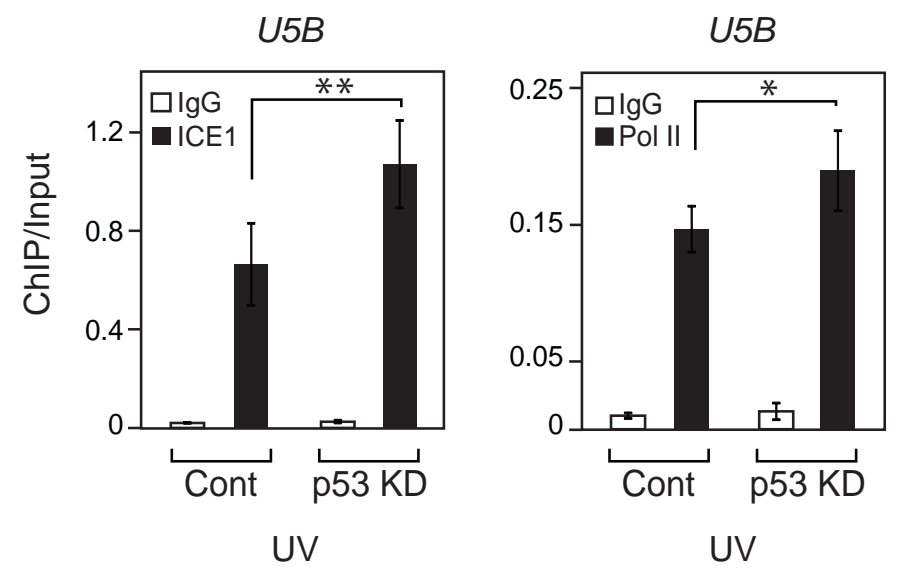

Figure 5 


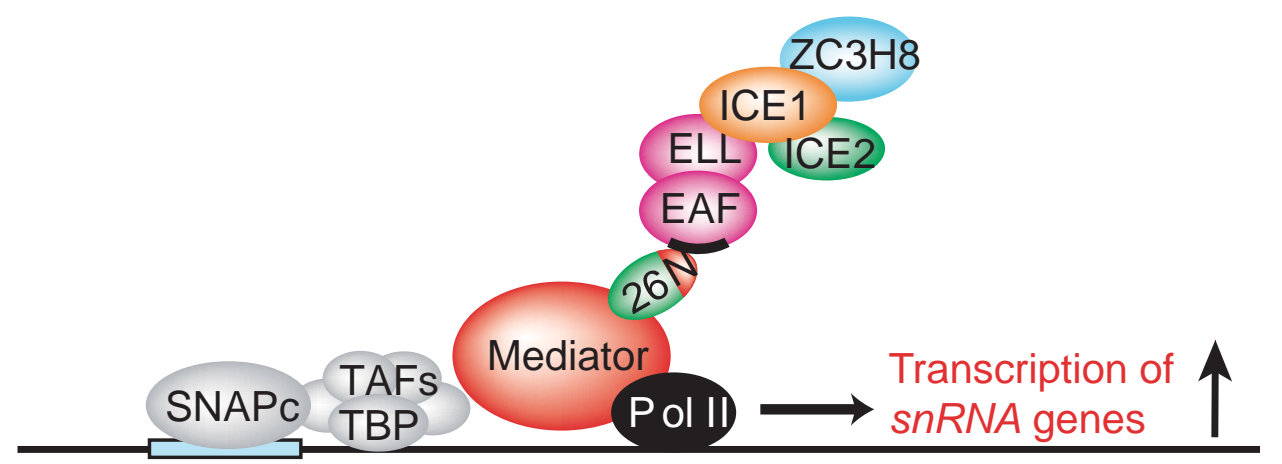

Promoter (PSE)
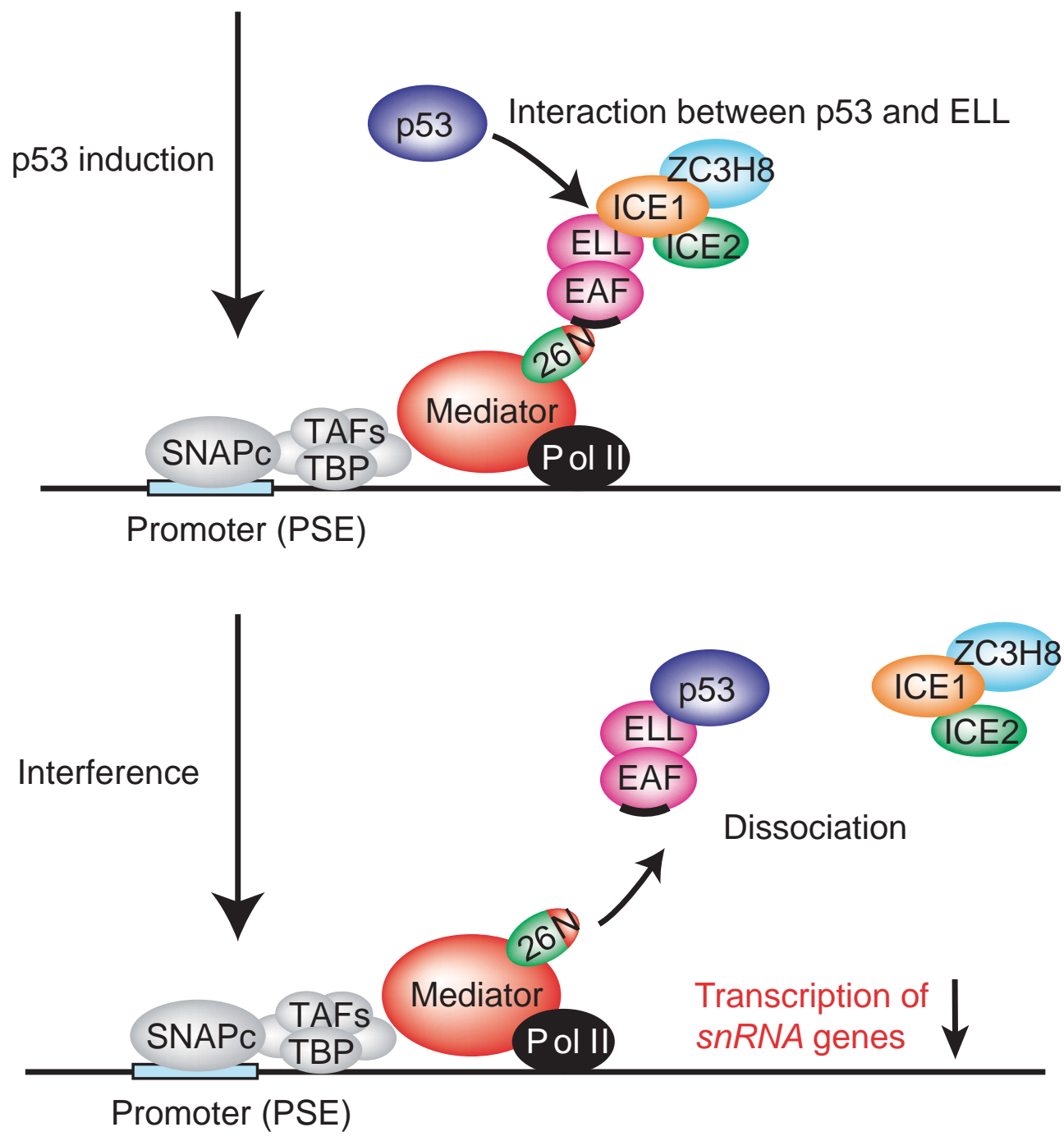

Figure 6 


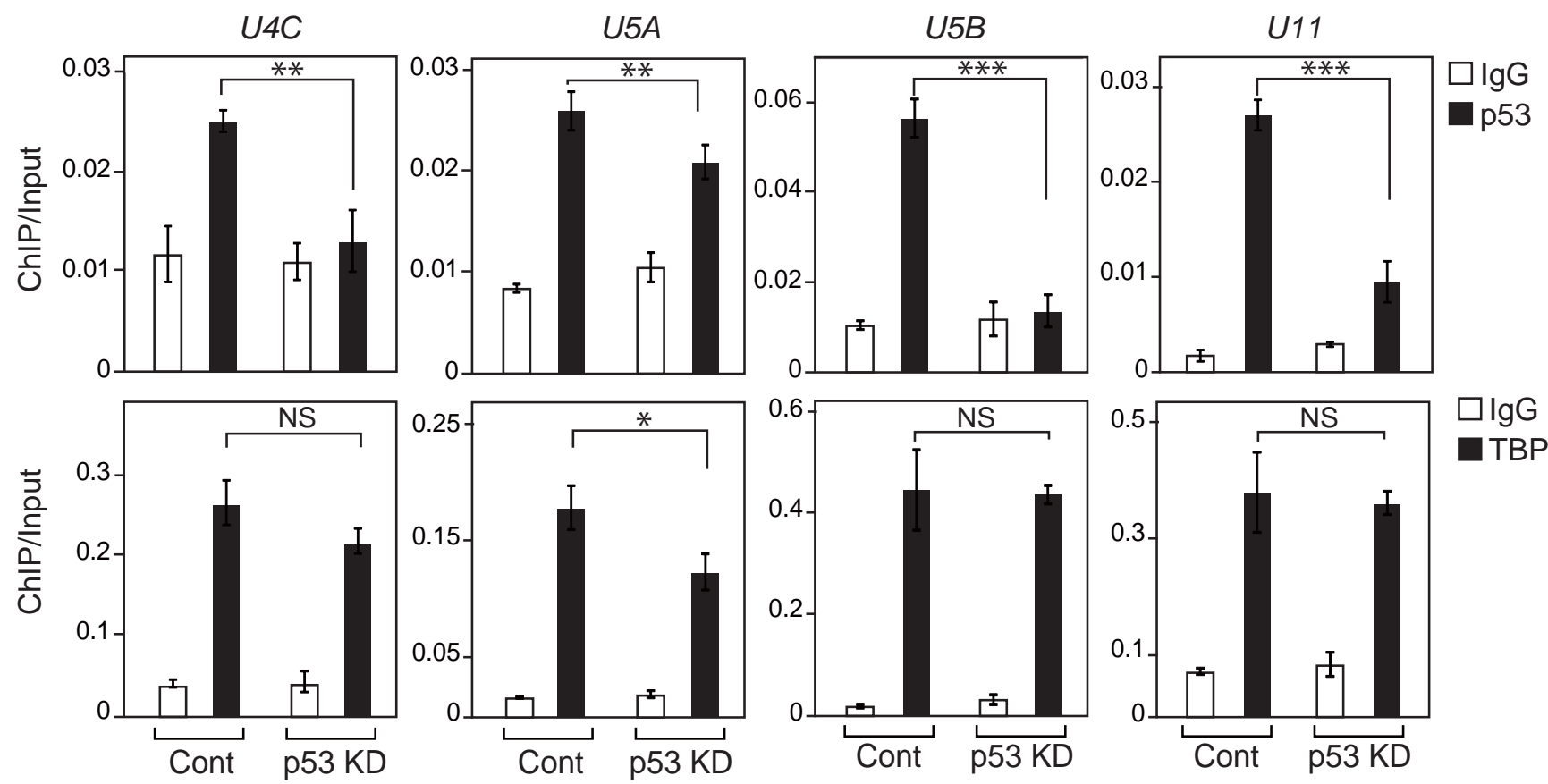

Supplementary Fig. 1. Knockdown of p53 decreases the occupancy of p53 at Pol II-dependent $s n R N A$ genes. The occupancy of p53 and TBP at the snRNA genes, including $U 4 C, U 5 A, U 5 B$ and U11 snRNA genes, was analyzed using control and p53-knockdown (KD) cells. Standard deviations of each result were calculated from three independent experiments. $P$ values were determined by Student's $t$ test $(* * * P<0.01$, ** $P<0.05)$. Error bars show s.d. NS, not specific. 

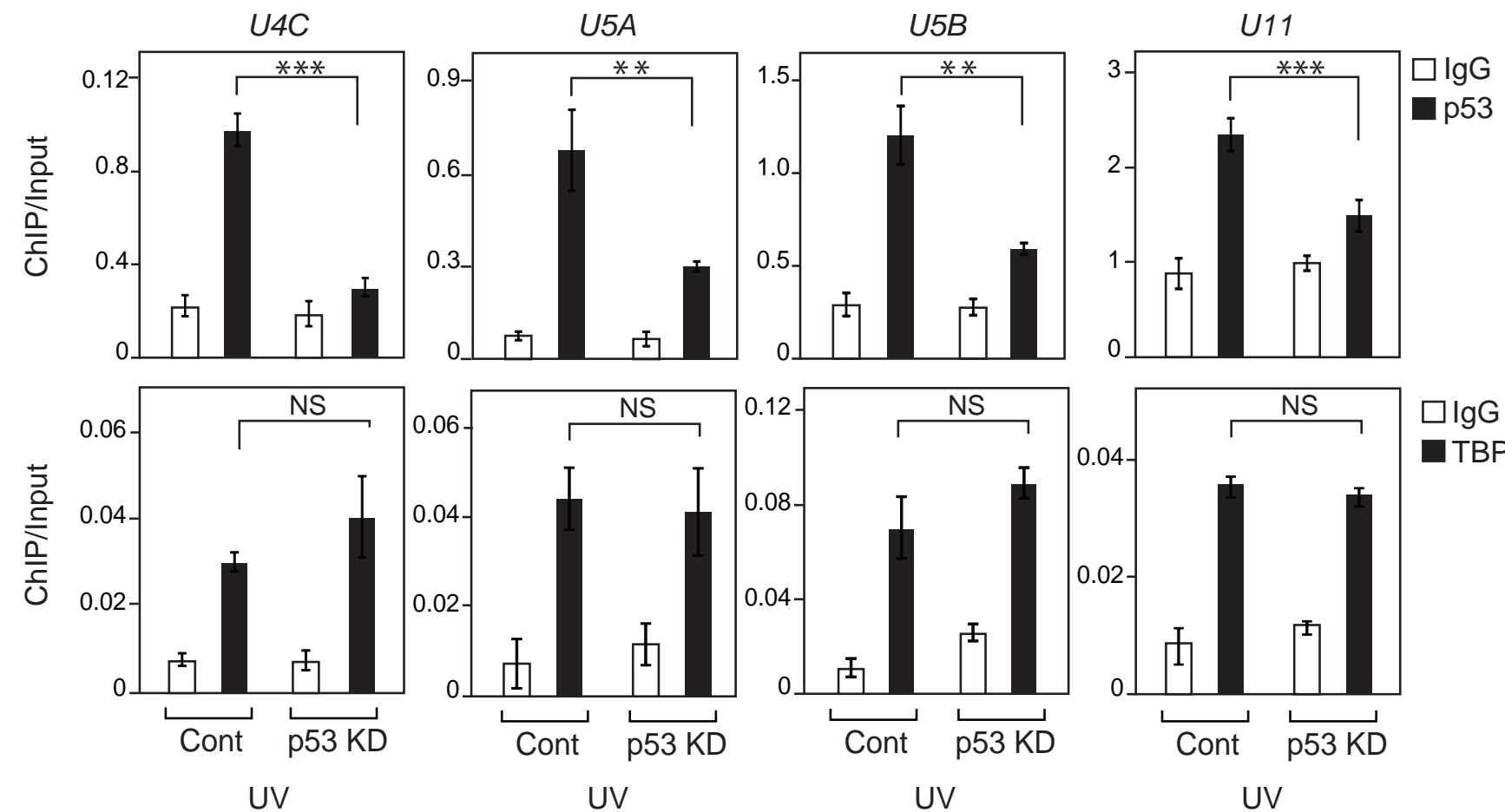

Supplementary Fig. S2. Knockdown of p53 decreases the occupancy of p53 after UV irradiation. After UV irradiation, the occupancy of p53 and TBP at snRNA genes, including U4C,U5A, U5B and U11 snRNA genes, was analyzed using control and p53-knockdown (KD) cells. Standard deviations of each result were calculated from three independent experiments. $P$ values were determined by Student's $t$ test $(* * * P<0.01$, ** $P<0.05)$. Error bars show s.d. NS, not specific. 


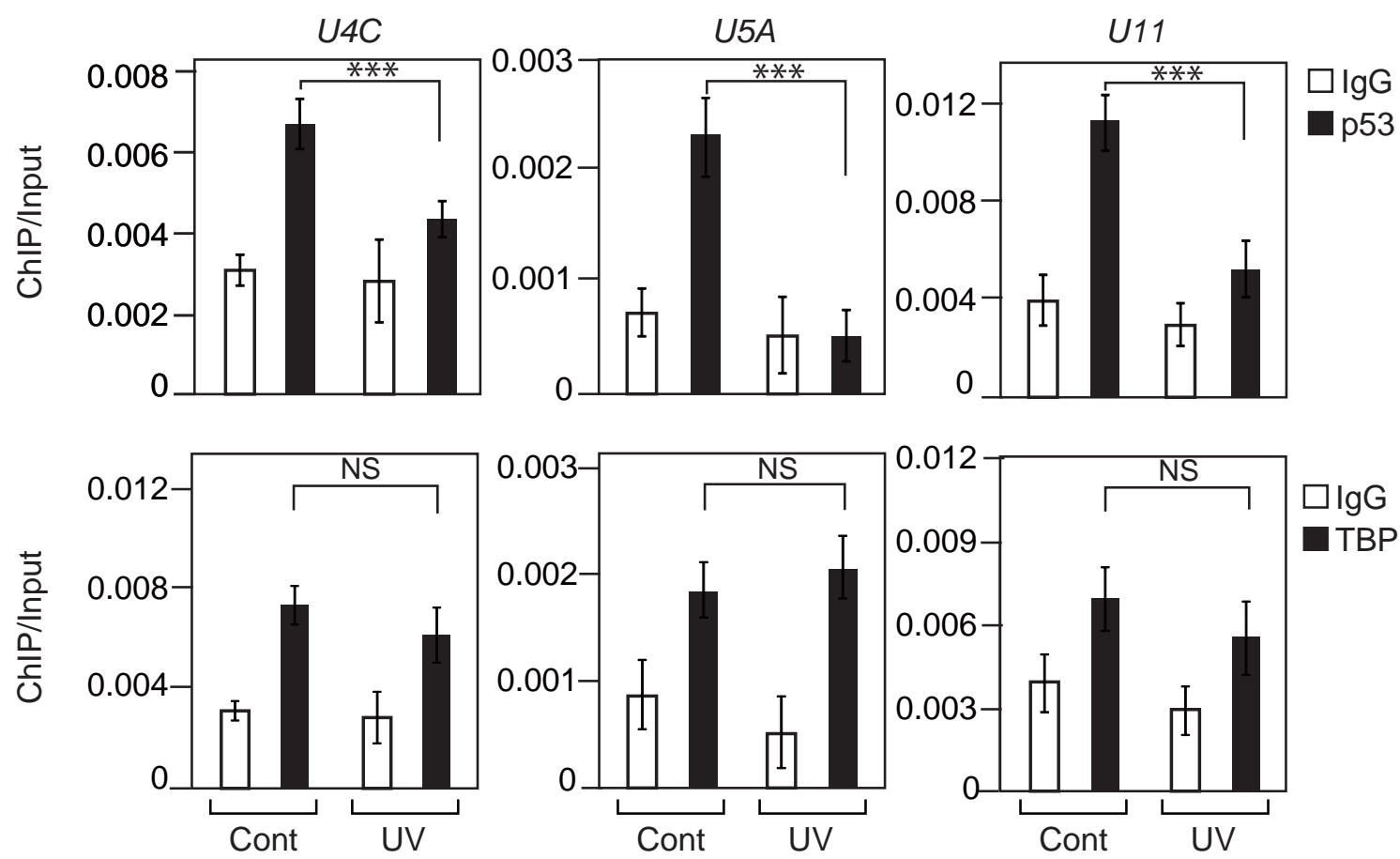

Supplementary Fig. S3. UV irradiation decreases the occupancy of p53 at snRNA genes. ChIP assays were performed using cells treated with or not treated with UV irradiation. The occupancy of p53 and TBP at snRNA genes, including $U 4 C, U 5 A$ and $U 11$ snRNA genes, was quantified after UV irradiation. Standard deviations of each result were calculated from three independent experiments. $P$ values were determined by Student's $t$ test $(* * * P<0.01)$. Error bars show s.d. NS, not specific. 